\title{
Early discrimination of nasopharyngeal carcinoma based on tissue deoxyribose nucleic acid surface-enhanced Raman spectroscopy analysis
}

\author{
Sufang Qiu \\ Chao Li \\ Jinyong Lin \\ Yuanji Xu \\ Jun $\mathrm{Lu}$ \\ Qingting Huang \\ Changyan Zou \\ Chao Chen \\ Nanyang Xiao \\ Duo Lin \\ Rong Chen \\ Jianji Pan \\ Shangyuan Feng
}




\title{
Early discrimination of nasopharyngeal carcinoma based on tissue deoxyribose nucleic acid surface-enhanced Raman spectroscopy analysis
}

\author{
Sufang Qiu, a,b,t Chao Li, ${ }^{c,+}$ Jinyong Lin, ${ }^{a}$ Yuanji Xu, ${ }^{c}$ Jun Lu, ${ }^{a}$ Qingting Huang, ${ }^{a}$ Changyan Zou, ${ }^{d}$ Chao Chen, ${ }^{d}$ \\ Nanyang Xiao, ${ }^{e}$ Duo Lin, ${ }^{f, g}$ Rong Chen, Jianji Pan, ${ }^{a, b, *}$ and Shangyuan Feng, \\ ${ }^{a}$ Fujian Cancer Hospital, Fujian Medical University Cancer Hospital, Department of Radiation Oncology, No. 420, Fuma Road, \\ Fuzhou 350014, Fujian, China \\ ${ }^{\mathrm{b}}$ Fujian Provincial Key Laboratory of Translational Cancer Medicine, Fuzhou, Fujian 350122, China \\ ${ }^{\mathrm{C} T}$ The Shengli Clinical College of Fujian Medical University, Fuzhou, Fujian 350001, China \\ ${ }^{\mathrm{d} F u j i a n}$ Provincial Cancer Hospital, Fujian Medical University Cancer Hospital, Department of Radiation Biological Laboratory, Fuzhou, \\ Fujian 350014, China \\ eFujian Normal University, The Key Laboratories of Innate Immune Biology of Fujian Province, Southern Biomedical Research Center, \\ Fuzhou 350007, China \\ ${ }^{f}$ Fujian Normal University, Fujian Provincial Key Laboratory for Photonics Technology, Key Laboratory of Optoelectronic Science and Technology \\ for Medicine, Ministry of Education, Fuzhou 350007, China \\ ${ }^{9}$ Fujian University of Traditional Chinese Medicine, College of Integrated Traditional Chinese and Western Medicine, Fuzhou 350108, China
}

\begin{abstract}
Surface-enhanced Raman spectroscopy (SERS) was employed to detect deoxyribose nucleic acid (DNA) variations associated with the development of nasopharyngeal carcinoma (NPC). Significant SERS spectral differences between the DNA extracted from early NPC, advanced NPC, and normal nasopharyngeal tissue specimens were observed at $678,729,788,1337,1421,1506$, and $1573 \mathrm{~cm}^{-1}$, which reflects the genetic variations in NPC. Principal component analysis combined with discriminant function analysis for early NPC discrimination yielded a diagnostic accuracy of $86.8 \%, 92.3 \%$, and $87.9 \%$ for early NPC, advanced NPC, and normal nasopharyngeal tissue DNA, respectively. In this exploratory study, we demonstrated the potential of SERS for early detection of NPC based on the DNA molecular study of biopsy tissues. $\odot 2016$ Society of PhotoOptical Instrumentation Engineers (SPIE) [DOI: 10.1117/1.JBO.21.12.125003]
\end{abstract}

Keywords: surface-enhanced Raman spectroscopy; nasopharyngeal carcinoma; deoxyribose nucleic acid; principal component analysis-discriminant function analysis.

Paper 160576RR received Aug. 25, 2016; accepted for publication Nov. 21, 2016; published online Dec. 9, 2016.

\section{Introduction}

Nasopharyngeal carcinoma (NPC) is one of the most commonly diagnosed head and neck cancers in southern China and Southeast Asia, with a reported incidence of $15-40$ per 100,000 population in endemic areas. ${ }^{1,2}$ With advances in radiotherapy, patients with early stage (stages I and II) NPC tend to achieve satisfactory treatment outcomes with an associated 5-year survival of up to $94 \%$ compared with $50 \%$ to $60 \%$ in patients diagnosed at advanced stages (stages III and IV). ${ }^{3,4}$ Therefore, early diagnosis plays a vital role in the treatment of patients with NPC. The symptoms of NPC in early stages are not obvious; ${ }^{5}$ pathological examination of the biopsy specimen is the gold standard for diagnosis. However, early stage NPC often occurs in the pharyngeal recess where it is hard to detect. Moreover, the size of the tumor in the early stages is often small; some of these are submucosal, which makes it challenging to obtain a biopsy on pharyngorhinoscopy. ${ }^{6}$ An inadequate specimen may also obscure the diagnosis.

Deoxyribose nucleic acid (DNA) is subject to modifications that are associated with evolution of genetic diseases or cancer. NPC is a genetic disease, and several biological characteristics

*Address all correspondence to: Jianji Pan, E-mail: panjianji@aliyun.com; Shangyuan Feng, E-mail: syfeng@fjnu.edu.cn

†These authors are cofirst authors and they contributed equally to the work. of cancer may be determined by $\mathrm{DNA}^{7}$ and related with EB-DNA, which can accelerate the proliferation of cancer cells and strengthen the cells more invasive and metastatic. ${ }^{8,9}$ Therefore, genetic testing may help in early detection of NPC. The Raman spectrum, also referred to as the "molecular fingerprint," ${ }^{10}$ can detect scattered photons with a spectrum of Raman peaks, each of which is characterized by a specific molecular bond. Collectively, these peaks provide intrinsic information of the sample and may yield a wealth of information about the chemical bonds associated with DNA and ribonucleic acid. Therefore, Raman spectroscopy technology can help in the early diagnosis of NPC by discriminating from the NPC tissues' DNA. Furthermore, the weak Raman signals in conventional Raman spectroscopy can be dramatically enhanced to $10^{6}$ to $10^{15}$ times by surface-enhanced Raman spectroscopy (SERS) technology when the analytes of interest are in close proximity to gold or silver nanoparticles, which could allow for ultrasensitive discrimination of biological molecules between different samples. ${ }^{11}$ Kneipp et al. ${ }^{12}$ reported that SERS can be a single molecule tool for bioanalytics.

Our group previously published preliminary findings on DNA variations in the saliva, blood, and tissues from patients with NPC by SERS. ${ }^{13-15}$ However, the interference of other native constituents and exogenous substances in untreated

$1083-3668 / 2016 / \$ 25.00$ @ 2016 SPIE 
Table 1 Specimen characteristics.

\begin{tabular}{lccc} 
& Normal group & Early NPC & Advanced NPC \\
\hline Number of samples & 30 & 26 & 35 \\
Mean age, years & 47 & 46 & 50 \\
$\begin{array}{l}\text { Mean DNA } \\
\text { concentration }\end{array}$ & $260 \mathrm{ng} / \mu \mathrm{L}$ & $280 \mathrm{ng} / \mu \mathrm{L}$ & $310 \mathrm{ng} / \mu \mathrm{L}$ \\
Clinical diagnosis & Normal & Stages I-II & Stages III-IV \\
\hline
\end{tabular}

saliva, blood, and tissue samples compromised the diagnostic accuracy of NPC using SERS and overwhelmed most DNA signals. To capture more DNA signatures for genetic testing, use of purified DNA for SERS analysis may be an ideal alternative for improving the early diagnosis of NPC.

At present, the application of purified DNA in the diagnosis of NPC has not been reported in the literature. In this work, we sought to use DNA SERS for discrimination between early stage NPC, advanced stage NPC, and normal tissues at the molecular level.

\section{Materials and Methods}

\subsection{Subjects and Protocol}

A total of 91 tissue DNA samples (from 26 early stage NPC patients, 35 advanced stage NPC patients, and 30 healthy volunteers) were used in the study (Table 1). All patients were confirmed cases of NPC on histopathology. The disease staging was based on the seventh edition of the International Union Against Cancer/American Joint Committee on Cancer staging system for NPC. ${ }^{16,17}$ The study was approved by the Fujian Provincial Tumor Hospital Ethics Committee (Reference No. FJCH-09911).

Tissue specimens obtained on pharyngorhinoscopy were kept in liquid nitrogen for half an hour. DNA extraction was performed according to the Takara manufacturer's protocol as previously described by Paterson et al. ${ }^{18}$ A schematic illustration of the preparation of DNA samples is shown in Fig. 1(a).
Ultimately, DNA was purified in distilled water, and the concentration was detected by biomedical analyzer. Before SERS measurement, the DNA solutions were stored in a $-20^{\circ} \mathrm{C}$ refrigerator to restrain degradation.

\subsection{Preparation of Silver (Ag) Colloids and DNA Mixture}

Ag colloids were prepared using hydroxylamine hydrochloride and $\mathrm{Ag}$ nitrate, in accordance with the method reported by Leopold and Lendl. ${ }^{19}$ In short, a total of $9 \mathrm{~mL}$ of $0.1 \mathrm{M}$ sodium hydroxide solution was mixed with $10 \mathrm{~mL}$ of $0.06 \mathrm{M}$ hydroxylamine hydrochloride solution to adjust the $\mathrm{pH}$ value of hydroxylamine hydrochloride solution. Then, the mixture was quickly added to $180 \mathrm{~mL}$ of $0.0011 \mathrm{M} \mathrm{Ag}$ nitrate solution, and the resulting mixture was shaken to obtain a homogeneous milky gray mixture. Absorption spectroscopy was utilized to characterize the prepared $\mathrm{Ag}$ colloids. The measurement demonstrated an absorption maximum at $418 \mathrm{~nm}$. The particle sizes were determined by transmission electron microscopy, as described in our previous paper. ${ }^{20}$ The particle sizes followed a normal distribution with a mean diameter of $35 \mathrm{~nm}$ and a standard deviation of $5 \mathrm{~nm}$. Next, the Ag colloids were concentrated by centrifugation at $10,000 \mathrm{rpm}$ for $10 \mathrm{~min}$ to get high-concentration Ag NPs as the SERS substrate. Before being subjected to SERS measurement, the DNA solution (about $250 \pm 50 \mathrm{ng} / \mu \mathrm{L}$ ) was mixed with silver colloid (about $2000 \mathrm{ng} / \mu \mathrm{L})$ (at a 1:1 ratio). Finally, a fixed amount $(2 \mu \mathrm{L})$ of DNA-silver colloid solution was dropped onto an aluminum (Al) plate for SERS measurement.

\subsection{SERS Measurement}

All SERS measurements were performed on a confocal microspectrometer equipped with a peltier cooled charge coupled device camera for spectral detection and a $785 \mathrm{~nm}$ diode laser for Raman excitation. A Leica $20 \times$ objective was used to collect the SERS signals from the sample illuminated by $1 \mathrm{~mW}$ of incident laser power. The SERS spectra were measured in the wave number range of 500 to $1700 \mathrm{~cm}^{-1}$. Each specimen was measured several times in different locations. The spectral acquisition and analysis were conducted using the package WIRE 2.0 (Renishaw plc).

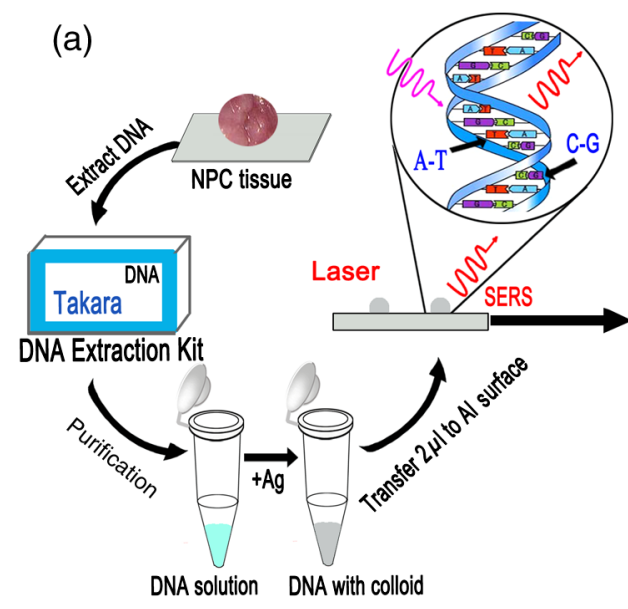

(b)

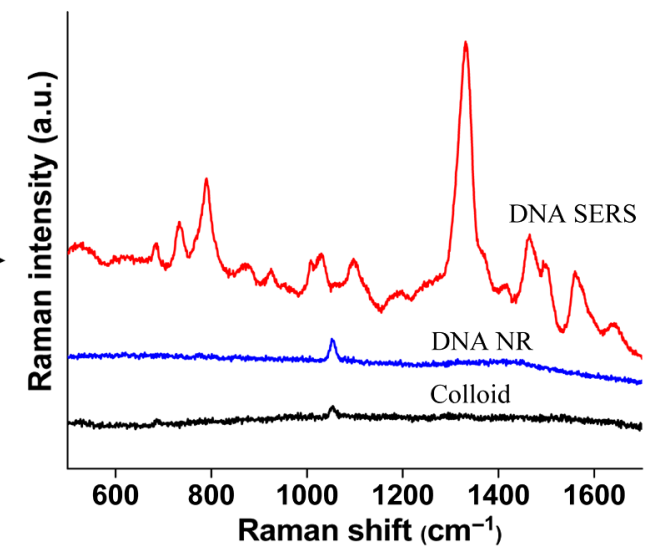

Fig. 1 (a) The schematic diagram of the preparation procedure of DNA-Ag NPs mixture. (b) Spectra taken from the DNA-Ag NPs mixture, the DNA sample without Ag NPs, and the Ag NPs substrate. 

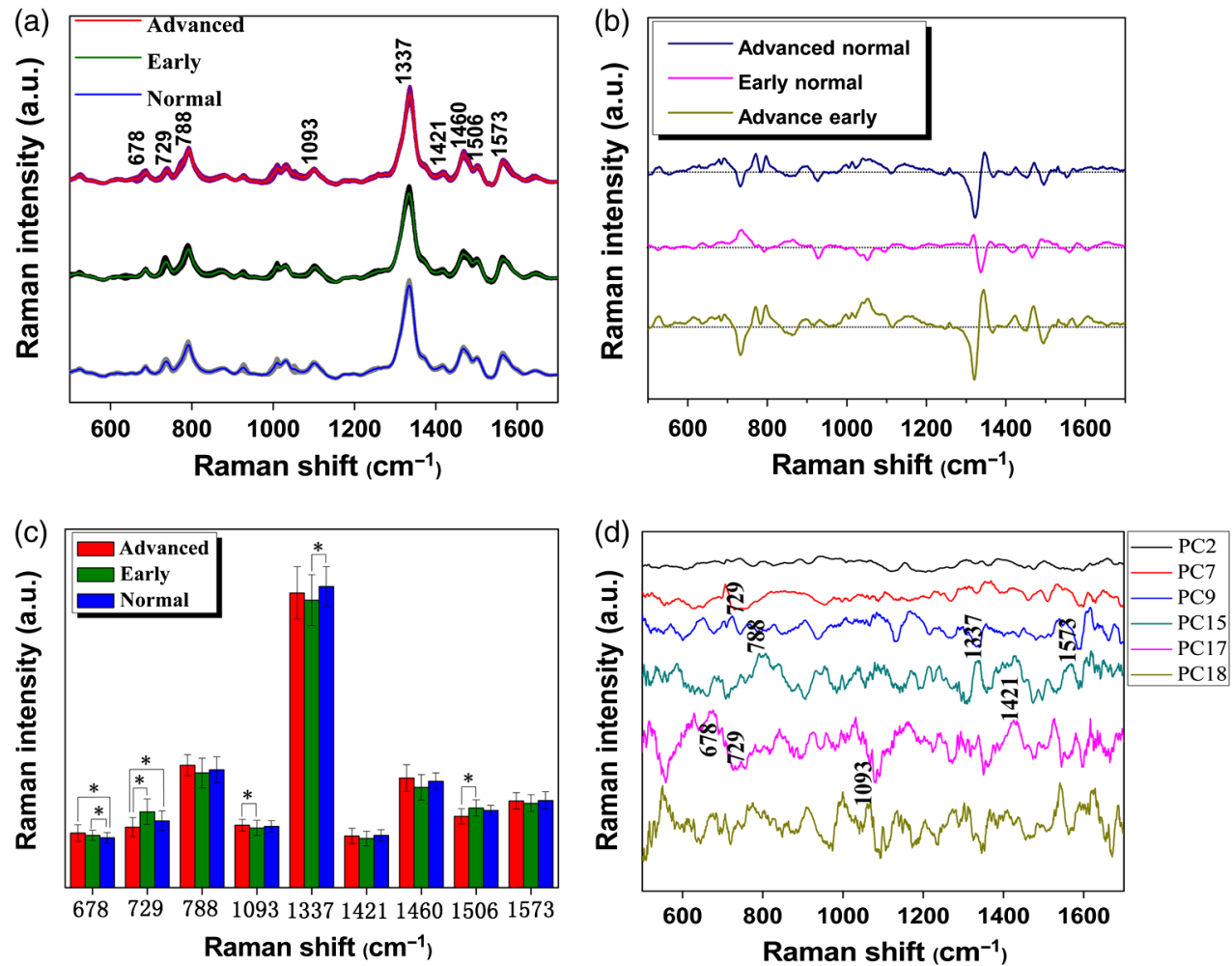

Fig. 2 (a) Comparison of the mean normalized SERS spectra of purified DNAs for the advanced stage NPC samples (red line; $n=35$ ), the early stage NPC DNA samples (green line; $n=26$ ), and the normal nasopharyngeal tissues samples (purple line; $n=30$ ). (b) Difference spectra were calculated from the mean SERS spectra among the three DNA types. (c) The histogram of three DNA types. (d) The six most diagnostically significant PC loadings plot (PC2, PC7, PC9, PC15, PC17, and PC18) $(P<0.05)$.

\subsection{Data Preprocessing}

In order to deduct background and to acquire the pure DNA SERS signal, the original spectral data were preprocessed using a five-order polynomial fitting algorithm. ${ }^{21}$ After fitting, area-under-the-curve normalization is applied to each spectrum to correct for variations in spectral absolute intensity. ${ }^{22}$

\subsection{PCA-DFA Analysis}

Efficient diagnostic algorithms for differentiating SERS spectra between the early NPC, advanced NPC, and normal nasopharyngeal tissue DNA were applied using principal component analysis (PCA) combined with discriminant function analysis (DFA) by SPSS 15.0 software package (SPSS Inc., Chicago). PCA was used to reduce the dimensionality of spectral data to several principal components (PCs). A dependent-sample $t$ test was conducted to select the most diagnostically significant PCs $(P<0.05)$. DFA was performed based on these diagnostically significant PCs to maximize between-group variance and minimize within-group variance. In this way, one sample (i.e., one spectrum) was excluded from the dataset, and the algorithm based on PCA-DFA was redeveloped using the remaining DNA spectra. To further evaluate the performance of the PCA-DFA algorithm, receiver operating characteristic (ROC) curves were generated.

\section{Results and Discussion}

Figure 1(b) shows the DNA SERS spectra after silver colloid enhancement. The DNA SERS peaks were abundant while the normal Raman spectral peaks were few due to the interference of large autofluorescence background. Meanwhile, the interference from silver colloid substrate was scarcely seen. The observations indicated that there were strong interactions between the silver nanoparticles and the DNA molecule. Because of this interaction, a DNA sample can closely adsorb on the silver colloid particles' surfaces, leading to quench biological autofluorescence and simultaneously increasing the intensity of the DNA Raman scattering. This is in agreement with the principle by Dulkeith et al. ${ }^{23}$

Raman bands, also referred to as "molecular fingerprints," can identify a particular chemical bond or a single functional group in the molecule. ${ }^{15,20}$ Therefore, the more abundant spectral peak signals emerged, the more the material component was reflected. In this study, prominent SERS peaks were observed in all 91 samples at 678, 729, 788, 1093, 1337, 1421, 1460, 1506, and $1573 \mathrm{~cm}^{-1}$ [Fig. 2(a)]. All SERS peaks were assigned to DNA according to the literature ${ }^{24,25}$ listed in Table 2.

Figures 2(b) and 2(c) show that the major SERS spectral differences between the DNA of normal, early, and advanced cancer tissue had different intensities of the bands. For example, the modes at $678,729,788,1337,1421,1506$, and $1573 \mathrm{~cm}^{-1}$ were due to DNA bases (adenine, thymine, cytosine, and guanine); $1093 \mathrm{~cm}^{-1}$ was attributed to the symmetric $\mathrm{PO}_{2}^{-}$stretching vibration of the DNA; and $1460 \mathrm{~cm}^{-1}$ was assigned to $\mathrm{CH}_{2} / \mathrm{CH}_{3}$ deformation of deoxyribose. It is common knowledge that carcinogenesis is prominently associated with DNA damage, especially via nucleobase lesions formed by reactive oxygen species. Meanwhile, a slight modification in the DNA can affect the intensity of Raman vibrations in the DNA 
Table 2 The tentative assignment of prominent SERS peaks observed in DNA samples.

Raman

shift $\left(\mathrm{cm}^{-1}\right)$

Assignment $^{\mathrm{a}}$

678

$G$ (ring breathing modes in the DNA bases)

729

788

A

1093

C, T

1337

1421

1460

1506

1573

aNote: A, adenine; T, thymine; C, cytosine; and G, guanine.

molecules. ${ }^{26}$ Malins et al. ${ }^{27}$ and Kourkoumelis et al. ${ }^{28}$ had reported that the intensity of spectral peak in 1093 and $1460 \mathrm{~cm}^{-1}$, respectively, were different between the normal cells and the cancer cells. These changes probably reflect the variations in relative DNA content and conformations that are involved in the occurrence and development of NPC.

Genomic instability is the hallmark of human cancer, being responsible for abnormal cancer cell proliferation, invasion, and metastasis. ${ }^{29}$ Many genetic mutations have been linked to pathogenesis of NPC, ${ }^{30}$ such as those linked to Epstein-Barr virus (EBV) infections. ${ }^{9}$ There exists some potential mechanisms that may explain the spectral differences among normal tissues, early stage, and advanced stage NPC tissues. For early stage NPC, lower intensities of most SERS peaks may be due to high frequency of loss of heterozygosity in the allelic status. ${ }^{31,32}$ With the progression of NPC, there was still a lack of heterozygosity on chromosome in advanced stage NPC. ${ }^{33}$ For advanced stage NPC, it was also found to be infected by EBV because of its high-grade dysplastic lesions, while EBV was hardly found in low-grade dysplastic lesions and the histologically normal nasopharyngeal epithelia. ${ }^{33}$ These variates may cause the differences among the three types of tissue DNAs. However, the other mechanisms for these spectra intensity differences of SERS peaks between normal, early stage, and advanced stage NPC warrant further investigation.

To quantitatively analyze the ability of DNA SERS technique for NPC detection, PCA-DFA was performed on the obtained SERS spectra. Forty-nine PCs (99\% of the total explained variance) were extracted by PCA. Then, the most diagnostically significant PCs (i.e., PC2, PC7, PC9, PC15, PC17, and PC18) $(P<0.05)$ were determined by the dependent-sample $t$ test. Figure 2(d) shows the plot of these six PC loadings. The plot enables one to know which spectral variables are dominating or influencing the PCA-DFA model. As the most diagnostically significant PCs, the prominent Raman peaks that appeared in these PC loadings, such as 678, 729, 788, 1093, 1337, 1421, and $1573 \mathrm{~cm}^{-1}$, are considered as the diagnostically relevant spectral bands. These bands are also easily identifiable in the difference spectrum [Fig. 2(b)]. Next, DFA was used to discriminate among the three groups based on these six PCs and a priori knowledge of which spectra were replicates, a process that does not bias the analysis.

Figure 3(a) shows the scatter plot of the coefficient for each sample according to the two discriminant functions after PCA for normal, early stage, and advanced stage NPC samples. The three scatter plots were distributed in three relatively separate areas in spite of the few overlaps, which indicates that the SERS spectra of the three different types of DNA samples could be well discriminated. Ultimately, use of the PCA-DFA algorithm for differentiation of DNA of normal, early stage NPC, and advance stage NPC achieved higher diagnostic sensitivities of $83.3 \%, 80.8 \%$, and $85.7 \%$; specificities of $83.6 \%$, $84.6 \%$, and $82.1 \%$; and accuracies of $87.9 \%, 86.8 \%$, and $92.3 \%$, respectively (Table 3 ).

We have done direct Raman spectral detection of tissue, ${ }^{34}$ which provided only a few characteristic peaks related to DNA, because most DNA signals were overwhelmed by other native constituents and exogenous substances in untreated tissue samples. DNA extraction and detection by SERS can capture more DNA fingerprints for improving the early diagnosis of NPC. In our study, the extracted DNA SERS spectra achieved
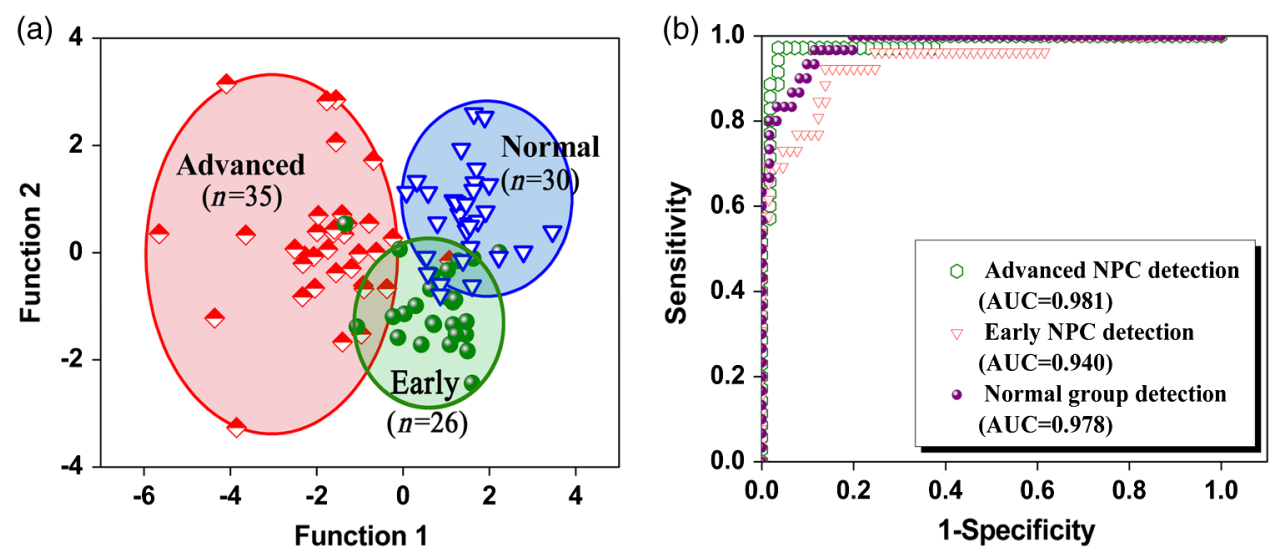

Fig. 3 (a) Scatter plot of the PCA-DFA demonstrating the clustering of DNAs from advanced stage, early stage, and normal nasopharyngeal tissue samples. (b) ROC curves of discrimination results generated from the PCA-DFA analysis. Note: The integrated AUCs are $0.981,0.940$, and 0.978 , respectively, for the three DNA groups. 
Table 3 Classification results of SERS spectra prediction of three DNA types using PCA-DFA.

\begin{tabular}{lccc} 
& \multicolumn{3}{c}{ Raman prediction } \\
\cline { 2 - 4 } DNA type & Normal group & Early NPC & Advanced NPC \\
\hline Normal & 25 & 5 & 0 \\
Early NPC & 3 & 21 & 2 \\
Advanced NPC & 3 & 2 & 30 \\
Sensitivity & $83.3(25 / 30)$ & $80.8(21 / 26)$ & $85.7(30 / 35)$ \\
Specificity & $83.6(55 / 61)$ & $84.6(58 / 65)$ & $82.1(54 / 56)$ \\
Accuracy & $87.9(80 / 91)$ & $86.8(79 / 91)$ & $92.3(84 / 91)$ \\
\hline
\end{tabular}

$86.8 \%$ of accuracy, superior to the results of $75 \%$ accuracy provided by direct tissue Raman spectra for early NPC detection. ${ }^{34}$

To further evaluate the performance of the PCA-DFA algorithm, ROC curves were generated [Fig. 3(b)]. The integration areas under the ROC curves were $0.981,0.940$, and 0.978 for advance stage NPC versus normal tissues, for early stage NPC versus normal tissues, and for both early and advanced stage NPC versus normal tissues, respectively. These high ROC areas demonstrate the good performance of the PCA-DFA method based on DNA SERS spectra for promoting early diagnosis of NPC. Furthermore, the complete process of SERS detection for DNA of NPC tissue, including the additional step of DNA extraction, takes only 1 day, which is shorter than that of the direct pathological detection for NPC tumor tissue (usually needs 3 to 5 days). Consequently, it is hoped that early detection of NPC may be achieved by specific SERS DNA, such as a single EBV DNA molecule, to provide a specific, rapid, and accurate diagnosis of NPC at an early stage.

In conclusion, the different intensities of significant SERS peaks could be recognized between the early stage, advanced stage, and normal tissues samples, which could reflect gene variations in NPC. PCA-DFA multivariate analysis was performed to classify three types of DNA SERS data, with a diagnostic accuracy of $87.9 \%, 86.8 \%$, and $92.3 \%$ for the three tissue groups, respectively. This exploratory study first underlines the potential of the SERS technique for early molecular diagnosis of NPC.

Meanwhile, we also recognize that it is still a challenge to apply this DNA-based SERS to clinical applications due to some limitations. First, our study has not identified the specific spectra of the DNA of EBV and cannot provide the detailed construction information of DNA. Second, the tissue sample size is too small to comprehensively evaluate the reproducibility and accuracy of this detection strategy. Therefore, further research needs to be conducted to sensitively detect the specific base sequence of EB-DNA and to systematically verify the utility of the proposed method in real clinical application.

\section{Disclosures}

There is no competing interest in this work.

\section{Acknowledgments}

The present study was funded by the National Clinical Key Specialty Construction Program (Grant No. 61210016), Key
Clinical Specialty Discipline Construction Program of Fujian (Grant No. 61178090), People's Republic of China and Fujian Provincial Key Laboratory of Translational Cancer Medicine (Grant No. 61178083), the National Natural Science Foundation of China (Grant Nos. 61210016, 61178090, 61575043, and 61405036), the Provincial Natural Science Foundation (Grant Nos. 2015J01436 and 2016J01292), Science and Technology Project of Fujian Province (Grant No. WKJ-FJ-01), Fujian Province Health Commission Young and Middle-Aged Talent Training Project (Grant No. 2014ZQN-JC-6), and Program for Chang Jiang Scholars and Innovative Research Team in University (Grant No. IRT1115).

\section{References}

1. S. M. Cao, M. J. Simons, and C. N. Qian, "The prevalence and prevention of nasopharyngeal carcinoma in China," Chin. J. Cancer 30(2), 114-119 (2011)

2. M. Adham et al., "Nasopharyngeal carcinoma in Indonesia: epidemiology, incidence, signs, and symptoms at presentation," Chin. J. Cancer 31(4), 185-196 (2012).

3. A. W. M. Lee et al., "Treatment results for nasopharyngeal carcinoma in the modern era: the Hong Kong experience," Int. J. Radiat. Oncol. Biol. Phys. 61(4), 1107-1116 (2005).

4. Y. P. Mao et al., "A clinical verification of the Chinese 2008 staging system for nasopharyngeal carcinoma," Chin. J. Cancer 28(10), 1022-1028 (2009)

5. A. W. Lee et al., "Nasopharyngeal carcinoma: presenting symptoms and duration before diagnosis," Hong Kong Med. J. 3(4), 355-361 (1997).

6. W. I. Wei et al., "The efficacy of fiberoptic endoscopic examination and biopsy in the detection of early nasopharyngeal carcinoma," Cancer 67(12), 3127-3130 (1991).

7. D. Hanahan and R. A. Weinberg, "Hallmarks of cancer: the next generation," Cell 144(5), 646-674 (2011).

8. J. C. Lin et al., "Quantification of plasma Epstein-Barr virus DNA in patients with advanced nasopharyngeal carcinoma," N. Engl. J. Med. 350(24), 2461-2470 (2004).

9. S. W. Tsao et al., "The role of Epstein-Barr virus in epithelial malignancies," J. Pathol. 235(2), 323-333 (2015).

10. S. Wachsmann-Hogiu, T. Weeks, and T. Huser, "Chemical analysis in vivo and in vitro by Raman spectroscopy-from single cells to humans," Curr. Opin. Biotechnol. 20(1), 63-73 (2009).

11. M. Fleischmann, P. J. Hendra, and A. J. McQuillan, "Raman spectra of pyridine adsorbed at a silver electrode," Chem. Phys. Lett. 26(2), 163-166 (1974)

12. J. Kneipp, H. Kneipp, and K. Kneipp, "SERS - a single-molecule and nanoscale tool for bioanalytics," Chem. Soc. Rev. 37(5), 1052-1060 (2008).

13. S. Feng et al., "Saliva analysis combining membrane protein purification with surface-enhanced Raman spectroscopy for nasopharyngeal cancer detection," Appl. Phys. Lett. 104(7), 073702 (2014).

14. D. Lin et al., "Label-free blood plasma test based on surface-enhanced Raman scattering for tumor stages detection in nasopharyngeal cancer," Sci. Rep. 4, 4751 (2014).

15. S. Feng et al., "Gold nanoparticle based surface-enhanced Raman scattering spectroscopy of cancerous and normal nasopharyngeal tissues under near-infrared laser excitation," Appl. Spectrosc. 63(10), 1089-1094 (2009).

16. S. B. Edge and C. C. Compton, "The American Joint Committee on cancer: the 7th edition of the AJCC cancer staging manual and the future of TNM," Ann. Surg. Oncol. 17(6), 1471-1474 (2010).

17. J. Pan et al., "A comparison between the Chinese 2008 and the 7 th edition AJCC staging systems for nasopharyngeal carcinoma," Am. J. Clin. Oncol. 38(2), 189-196 (2015).

18. P. J. Paterson et al., "Development of molecular methods for the identification of aspergillus and emerging moulds in paraffin wax embedded tissue sections," Mol. Pathol. 56(6), 368-370 (2003).

19. N. Leopold and B. Lendl, "A new method for fast preparation of highly surface-enhanced Raman scattering (SERS) active silver colloids a 
room temperature by reduction of silver nitrate with hydroxylamine hydrochloride," J. Phys. Chem. B 107(24), 5723-5727 (2003).

20. S. Feng et al., "Nasopharyngeal cancer detection based on blood plasma surface-enhanced Raman spectroscopy and multivariate analysis," Biosens. Bioelectron. 25(11), 2414-2419 (2010).

21. J. Zhao et al., "Automated autofluorescence background subtraction algorithm for biomedical Raman spectroscopy," Appl. Spectrosc. 61(11), 1225-1232 (2007).

22. Z. Huang et al., "Near-infrared Raman spectroscopy for optical diagnosis of lung cancer," Int. J. Cancer 107(6), 1047-1052 (2003).

23. E. Dulkeith et al., "Fluorescence quenching of dye molecules near gold nanoparticles: radiative and nonradiative effects," Phys. Rev. Lett. 89(20), 111-118 (2002).

24. Z. Movasaghi, S. Rehman, and I. U. Rehman, "Raman spectroscopy of biological tissues," Appl. Spectrosc. Rev. 42(5), 493-541 (2007).

25. J. De Gelder et al., "Reference database of Raman spectra of biological molecules," J. Raman Spectrosc. 38(9), 1133-1147 (2007).

26. R. E. Dickerson and H. R. Drew, "Kinematic model for B-DNA," Proc. Natl. Acad. Sci. U. S. A. 78(12), 7318-7322 (1981).

27. D. C. Malins et al., "A cancer DNA phenotype in healthy prostates, conserved in tumors and adjacent normal cells, implies a relationship to carcinogenesis," Proc. Natl. Acad. Sci. U. S. A. 102(52), 19093-19096 (2005).

28. N. Kourkoumelis et al., "Advances in the in vivo Raman spectroscopy of malignant skin tumors using portable instrumentation," Int. J. Mol. Sci. 16(7), 14554-14570 (2015).

29. S. Negrini, V. G. Gorgoulis, and T. D. Halazonetis, "Genomic instability-an evolving hallmark of cancer," Nat. Rev. Mol. Cell Biol. 11(3), 220-228 (2010).

30. D. C. Lin et al., "The genomic landscape of nasopharyngeal carcinoma," Nat. Genet. 46(8), 866-871 (2014).

31. A. S. C. Chan et al., "High frequency of chromosome 3p deletion in histologically normal nasopharyngeal epithelia from southern Chinese," Cancer Res. 60(19), 5365-5370 (2000).

32. P. van der Riet et al., "Frequent loss of chromosome 9p21-22 early in head and neck cancer progression," Cancer Res. 54(5), 1156-1158 (1994).

33. K. W. Lo and D. P. Huang, "Genetic and epigenetic changes in nasopharyngeal carcinoma," Semin. Cancer Biol. 12(6), 451-462 (2002).

34. S. Qiu et al., "Non-invasive detection of nasopharyngeal carcinoma using saliva surface-enhanced Raman spectroscopy," Oncol. Lett. 11(1), 884-890 (2015).

Sufang Qiu received her MD degree from Fujian Medical University in 2016. She is an associate professor in the Radiation Oncology Department, Fujian Medical University Cancer Hospital, China. Her research interest focuses on head and neck cancer comprehensive diagnosis and therapy.

Chao $\mathbf{L i}$ is a postgraduate at Fujian Medical University, Fuzhou, Fujian. His research focuses on the diagnosis and research of nasopharyngeal carcinoma.
Jinyong Lin received his MS degree from Fujian Normal University. Currently, he is a medical physicist at Fujian Cancer Hospital. His research focuses on the application of Raman spectroscopy in biomedical diagnosis and cancer research.

Yuanji Xu is a MD at Fujian Medical University. His research interest focuses on head and neck cancer comprehensive diagnosis and therapy.

Jun Lu is a medical physicist at Fujian Cancer Hospital. His research interest focuses on radiation physics.

Qingting Huang received his MS degree from Fujian Medical University in 2016. His research focuses on the diagnosis and research of nasopharyngeal carcinoma.

Changyan Zou is an experimental technician at Fujian Cancer Hospital. His research focuses on radiation biology.

Chao Chen is an experimental technician at Fujian Cancer Hospital. His research focuses on radiation biology.

Nanyang Xiao is a MD at Fujian Normal University. Currently, he is an assistant at the Key Laboratories of Innate Inimune Biology of Fujian Province, Southern Biomedical Research Center, Fujian Normal University, Fuzhou.

Duo Lin received his master's degree in physical electronics from Fujian Normal University. Currently, he is an assistant at Fujian University of Traditional Chinese Medicine. His current research focuses on the biomedical detection using SERS.

Rong Chen is a professor at the School of Optoelectronics and Information Engineering, Fujian Normal University, China. His research interest focuses on the application of Raman spectroscopy in biomedical detection.

Jianji Pan received his MD degree from France Paris N0.2 University in 1994 . He is a professor at the Radiation Oncology Department, Fujian Medical University Cancer Hospital, China. His research interest focuses on head and neck cancer comprehensive diagnosis and therapy.

Shangyuan Feng received his PhD from Fujian Normal University in 2011. He started a postdoctoral position in BC Cancer Agency, Canada, in 2013. Currently, he is an associate professor at the School of Optoelectronics and Information Engineering, Fujian Normal University, China. His research interest focuses on the application of SERS in biomedical diagnosis. 